\title{
Small-angle scattering model for multilamellar vesicles
}

\author{
Henrich Frielinghaus \\ Jülich Centre for Neutron Science, Forschungszentrum Jülich GmbH, D-85748 Garching, Germany
}

(Received 19 July 2007; revised manuscript received 31 August 2007; published 21 November 2007)

\begin{abstract}
A small-angle neutron-X-ray-light-scattering model for multilamellar vesicles is developed on the basis of a simple geometry. $N$ spherical shells with radii of an arithmetic series are allowed for displacements $\Delta R$ which are limited by $\Delta R<R / N$ due to sterical reasons, with $R$ being the radius of the vesicle. The model shows many properties over a large $Q$ range which include a Guinier region, a first power law, a correlation peak, and a second power law connected to the surface properties of the bilayer. The first power law is related to the compactness of the vesicle and lies between $Q^{-2}$ for surfaces and $Q^{-4}$ for compact volumes (Porod law). The exact exponent is related to the number of shells $N$. The correlation peak has a maximum sharpness for rather small displacements $\Delta R$, but no second order peak is predicted. Only for rather large displacements the correlation peak widens up and shifts to smaller scattering angles. Then the important bilayer spacing is larger. The predictive power of the model lies in the connection of the compactness with $N$ and in the maximum correlation peak sharpness. This model considers many length scales at a time while existing theories focus on length scales of the bilayer spacing and the bilayer itself.
\end{abstract}

DOI: 10.1103/PhysRevE.76.051603

PACS number(s): 68.15.+e, 82.70.Uv, 87.16.Dg, 61.12.Ex

\section{INTRODUCTION}

Vesicles are formed by bilayers in solution (mainly in water) [1]. The bilayers in turn are formed by amphiphilic molecules such as surfactants or lipids, but also by amphiphilic diblock copolymers. Vesicles of the latter molecules are also called liposomes and polymersomes. The amphiphilicity leads to the formation of a hydrophobic bilayer core and the hydrophilic head groups point towards the water. In this sense the bilayer has a symmetric $A B A$ structure (hydrophilic-hydrophobic-hydrophilic). Just recently, it was found that symmetric $A B A$ triblock copolymers with a rigid midblock form vesicles equally well [2]. The rigidity of the midblock ensures that the peripheral blocks are at opposite sides of the layer.

The Helfrich model reduces the properties of the bilayer to two elastic moduli [3]. The bending rigidity $\kappa$ is connected with the mean curvature, while the saddle splay modulus $\bar{\kappa}$ is linked with the Gaussian curvature. The lamellar phase with an average mean curvature of zero is found for relatively large bending rigidities $\kappa$. Vesicles are always found close to the lamellar phase region, since the mean curvature is rather small [4]. They can be kinetically stable after applying shear on a lamellar phase [4-6]. Then, often multilamellar vesicles (MLV) form with many shells in one another, and these MLVs are rather densely packed. But even for diluted samples unilamellar and multilamellar vesicles appear $[1,4]$.

Unilamellar and multilamellar vesicles have been studied extensively by scattering methods and by real space imaging methods during the past years [1]. Since the overall dimension reaches often from several $10 \mathrm{~nm}$ to the $10 \mu \mathrm{m}$ scale, real space imaging methods such as atomic force and light microscopy are often successful. Static small angle light scattering reaches the same length scales, and thus gives insight about the overall size. Scattering experiments have the advantage that all considered parameters are obtained as average values with good statistics. Furthermore, the regular spacing of multilamellar vesicles is obtained by small angle neutron (SANS) and x-ray scattering (SAXS) experiments. The typical distances lie in the 10 to $100 \mathrm{~nm}$ region. Especially this internal structure is only resolved by scattering experiments. Most experiments aimed at the two different length scales independently, while technically light and neutron or x-ray scattering experiments could be combined to obtain a single scattering function, and even small angle neutron scattering instruments alone would allow for a continuous scattering curve over many length scales [7]. Small vesicles $(\sim 10 \mathrm{~nm})$ are observed over the full $Q$ range by conventional SANS experiments [8,9]. Larger unilamellar vesicles are also well described by SANS [10,11].

The modeling of scattering curves of unilamellar vesicles covers the overall properties down to simple properties of the bilayer [8]. It is assumed that the bilayer is homogeneous with respect to the scattering power and this bilayer forms a single spherical shell. In this respect the vesicle is treated as a hollow sphere with finite thickness. This model was applied successfully for small vesicles where the bilayer thickness is not much smaller than the vesicle diameter. A correlation peak accounts for the alternating structure bilayersolvent-bilayer. For larger unilamellar vesicles this correlation is not dominant anymore.

For multilamellar vesicles the correlation between different shells becomes important. This detail of the structure becomes observable by conventional SANS and SAXS experiments $[5,8]$. A first order correlation peak and sometimes higher order peaks tell about the spacing and correlations of the structure. The modeling assumes that the shells are locally flat and thus the MLV resembles a planar lamellar structure for these length scales. Membrane undulations are the major issue for disorder. The similarity to the lamellar phase is not unreasonable since the outer shells of MLVs are rather planar, and their contribution to the scattering is most important. The first scattering model for lamellar membranes was described by Caillé [12]. At this stage the model describes a perfectly aligned sample which was successfully applied by Ref. [13]. An extended theory described addi- 
tional long range order [14], which is well reviewed by Ref. [15] and brought to a final numerical solution including orientational averaging for powder samples. At this stage a real lamellar phase with multiple domains cannot be distinguished from a concentrated multilamellar vesicle phase.

Fluctuations of a unilamellar vesicle membrane are successfully described by spherical harmonics [16-19]. In principle, these models could be used for multilamellar vesicles if the independence of the fluctuating shells is assumed. This scenario will also be discussed in the discussion section. The disorder caused by translational motions, i.e., by displacements, is always present-even for rather rigid membranes. While the zeroth order of description of concentric shells is already published [2], displacements are a first order approach of disorder or fluctuations and are presented in this article. These initial approaches of small angle scattering models for MLVs are the first of its kind.

Since many multilamellar vesicles are obtained from a lamellar phase which is sheared and then kinetically locked the spacing of the sheets is extremely similar to the original lamellar phase $[5,8,20,21]$. So the amphiphile concentration is roughly given by $\phi \approx d / a$, with $d$ being the bilayer thickness and $a$ being the spacing of the shells. The spacing of the shells is given by the scattering vector of the first correlation peak, i.e., $a=2 \pi / Q$. Once the MLVs leave considerable space between each other then $\phi<d / a$ holds. This condition coincides with the assumption of dilution of the Guinier law. The Guinier law assumes that the concentration of solid particles is much smaller compared to unity. The ratio $d / a$ just takes care of the real internal bilayer mass of a MLV, and so the Guinier assumption reads $\phi \ll d / a$.

Experiments along the dilution line [22] proved that concentrated MLVs do not considerably change the shape upon dilution. Typically for concentrated MLVs many Bragg peaks are observed. Reference [22] especially uses the same nomenclature of form and structure factors as we will use throughout this paper.

The paper is organized in the following way. First a brief review summarizes the terms form and structure factor for MLVs. Then the structure factor of the shell formation in MLVs is described. The idea behind this derivation deals with exactly concentric spherical shells first, before displacements allow for some disorder. A simple bilayer form factor is introduced then and the absolute macroscopic cross section is derived. Numerical evaluations will be presented to show the richness of this model. The final discussion will discuss the development stage of the model.

\section{BRIEF REVIEW OF UNILAMELLAR VESICLES}

Unilamellar vesicles alone can already show a rich variety of scattering curves. The simplest model assumes that the scattering arises from a homogenous shell embedded in the solvent. The homogeneity means that the scattering length density stays constant within the shell of finite thickness. So the chemically distinct parts of the amphiphilic molecule are assumed to scatter with the same strength at the present state of the model. The scattering amplitude as a function of the scattering vector $Q$ is calculated according to

$$
\begin{aligned}
A(Q) & =\int_{\text {shell }} \Delta \rho(\vec{r}) \exp (i \vec{Q} \cdot \vec{r}) d^{3} r \\
& =4 \pi \int_{\text {shell }} \Delta \rho(r) r^{2} \frac{\sin (Q r)}{Q r} d r .
\end{aligned}
$$

The first line of Eq. (1) describes the scattering amplitude of a general structure ( $i$ being the imaginary unit) while the second line already makes use of the spherical symmetry. The scattering contrast $\Delta \rho(r)$ with respect to the solvent is a function of the absolute value of the vector $\vec{r}$ only. The factor $4 \pi r^{2}$ is the surface of the sphere at the considered radius $r$ and the factor $\sin (Q r) /(Q r)$ describes the integrated phase of an infinitely thin shell of radius $r$. The integration between the two limiting radii $R_{1}$ and $R_{2}$ still has to be carried out, and one finally arrives at

$$
\begin{aligned}
A(Q)= & (\Delta \rho)^{2} \frac{4 \pi}{3}\left(R_{2}^{3} f_{\mathrm{sph}}\left(Q R_{2}\right)-R_{1}^{3} f_{\mathrm{sph}}\left(Q R_{1}\right)\right) \\
& \text { with } f_{\mathrm{sph}}(x)=3 \frac{\sin (x)-x \cos (x)}{x^{3}} .
\end{aligned}
$$

The function $f_{\mathrm{sph}}(\mathrm{x})$ describes a solid sphere. Due to the difference in Eq. (2) a hollow sphere is obtained. In this simple model the macroscopic cross section of $n$ independent vesicles is obtained by the square of the amplitude and the normalization to the total volume $V$ :

$$
\begin{aligned}
\frac{d \Sigma}{d \Omega}(Q)= & \frac{n}{V}|A(Q)|^{2}=(\Delta \rho)^{2} \phi V_{\text {shell }} \\
& \times\left(\frac{(4 \pi / 3) R_{2}^{3} f_{\text {sph }}\left(Q R_{2}\right)-(4 \pi / 3) R_{1}^{3} f_{\text {sph }}\left(Q R_{1}\right)}{V_{\text {shell }}}\right)^{2} .
\end{aligned}
$$

The shell volume $V_{\text {shell }}=(4 \pi / 3)\left(R_{2}^{3}-R_{1}^{3}\right)$ is also used in the amphiphile concentration $\phi=n V_{\text {shell }} / V$. The last factor is the form factor of a shell with finite thickness. This model was used by Ref. [8].

While this description is exact in itself, another approach can be made to distinguish between the thin shell and the bilayer structure. For this purpose we write for the radius $r$ $=R+\delta$ and start from Eq. (1) again to obtain

$$
A(Q)=4 \pi R^{2} \frac{\sin (Q R)}{Q R} \int_{\text {shell }} \Delta \rho(R+\delta) \cos (Q \delta) d \delta .
$$

Equation (4) represents the leading term which becomes dominant for thin structures compared to the principal radius $R$. Now the macroscopic cross section can be written in a factorized way:

$$
\frac{d \Sigma}{d \Omega}(Q)=\phi V_{\text {shell }} S(Q) F(Q) .
$$

The volume of the shell reads now $V_{\text {shell }}=4 \pi R^{2} \Delta$ with $\Delta$ being the bilayer thickness. The structure factor $S(Q)$ $=[\sin (Q R) /(Q R)]^{2}$ describes the structure of an infinitely thin shell, whereas the form factor $F(Q)$ $=\left[\frac{1}{\Delta} \int \Delta \rho \cos (Q \delta) d \delta\right]^{2}$ contains the detailed information 
about the bilayer structure on a local scale (associated with $\delta)$. The major advantage of this factorization is the separation of the large scale structure expressed by $S(Q)$ and the small scale structure given by $F(Q)$. The spirit of this separation is transported by Pedersen [23].

Both Eqs. (3) and (5) describe unilamellar vesicles on all length scales. For large (giant) vesicles one finds three regions: The Guinier region describes the whole particle, a power law describes the surface properties of the shell, and at larger scattering angles-in principle-the compact volume properties of a bilayer are described. For small vesicles when the bilayer thickness is comparable to the shell diameter [only Eq. (3)] this picture breaks down and mainly a correlation peak appears [8]. The scattering of small vesicles accounts for bilayer-solvent-bilayer correlation across the particle. Nonetheless, the large vesicles are in the focus of the current paper.

\section{STRUCTURE FACTOR OF MULTILAMELLAR VESICLES}

In the following a structure factor for multilamellar vesicles shall be derived. It will idealize the single shells as infinitely thin, and the bilayer structure will be described by the form factor. The structure factor shall keep track of the appearance of the whole vesicle but it shall also describe the shell spacing at smaller length scales. As we will see in the discussion section, the whole concept is suited to describe a rich variety of properties. We start with the description of a single shell

$$
F_{j}(Q)=\frac{j^{2}}{N^{2}} \frac{\sin (Q R j / N)}{(Q R j / N)} .
$$

Each shell is numbered by an index $j$ running from 1 (innermost shell) to $N$ (outermost shell). So the MLV has $N$ shells with an arithmetic series of radii. The maximum radius is given by $R$. All single structure factors are normalized in that way, that the outermost contribution is unity at small scatter-

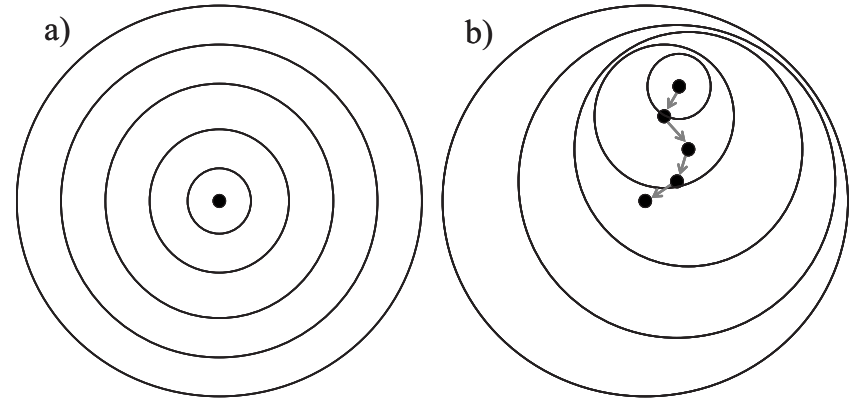

FIG. 1. The geometrical assembly of a multilamellar vesicle. On the left (a) all shells are concentric indicated by a single dot in the center. The shell radii are an arithmetic series, i.e., the shell radius $j$ has the size $j R / N$. On the right (b) the shells are additionally allowed to be shifted with respect to the predecessor. The shift vectors are called $\vec{v}_{j}$. The sequence of these vectors is a random walk.

ing angles $Q$ whereas the terms of the inner shells scale with the area being proportional to $j^{2}$. The current expression assumes all shells being concentric, since all contributions are real. For the total scattering amplitude all single contributions are summed up:

$$
A(Q)=1 F_{1}+e^{i \vec{Q} \cdot \vec{v}_{2}} F_{2}+e^{i \vec{Q}\left(\vec{v}_{2}+\vec{v}_{3}\right)} F_{3}+\cdots+e^{i \vec{Q}\left(\vec{v}_{2}+\cdots+\vec{v}_{N}\right)} F_{N} .
$$

Now displacements of the shell centers are included, which is indicated by the phases $e^{i \vec{Q} \cdot \vec{v}_{j}}$. The shift vector is given by $\vec{v}_{j}$. A sketch for the geometrical meaning of the amplitude is presented in Fig. 1. On the left the concentric case $\left(\vec{v}_{j}=\overrightarrow{0}\right)$ is depicted. On the right nonvanishing displacements are allowed. Due to sterical reasons of the rigid shells the maximum shift is given by $\left|\vec{v}_{j}\right| \leqslant \Delta R=R / N$. The series of $\vec{v}_{j}$ is describing a random walk as indicated in Fig. 1. The structure factor of a MLV is now defined as the square of the amplitude, and so it reads

$$
\begin{aligned}
S(Q)= & \frac{N^{2}}{\frac{1}{3} N\left(N+\frac{1}{2}\right)(N+1)}\left\langle|A|^{2}\right\rangle_{v_{2}, \ldots, v_{N}} \\
= & \frac{N}{\frac{1}{3}\left(N+\frac{1}{2}\right)(N+1)}\left(F_{1}^{2}+F_{2}^{2}+\cdots+F_{N}^{2}+2\left\langle\cos \left(\vec{Q} \vec{v}_{2}\right)\right\rangle_{v_{2}} F_{1} F_{2}\right. \\
& +2\left\langle\cos \left(\vec{Q} \vec{v}_{3}\right)\right\rangle_{v_{3}} F_{2} F_{3}+2\left\langle\cos \left(\vec{Q}\left(\vec{v}_{2}+\vec{v}_{3}\right)\right\rangle_{v_{2}, v_{3}} F_{1} F_{3}\right. \\
& \vdots \\
& +2\left\langle\cos \left(\vec{Q} \vec{v}_{N}\right)\right\rangle_{v_{N}} F_{N-1} F_{N}+\cdots+2\left\langle\cos \left(\vec{Q}\left(\vec{v}_{2}+\cdots+\vec{v}_{N}\right)\right\rangle_{v_{2}, \ldots v_{N}} F_{1} F_{N}\right) .
\end{aligned}
$$


The current structure factor is normalized in that way that the limit at small scattering vectors is the sum of all fractions of surfaces inside the MLV, i.e., $S(0)=\Sigma(j / N)^{2}$. In expression (8) one takes the statistics of the shift vectors into account. In the following it is simply assumed that they can take any value within the limiting sphere $\left|\vec{v}_{j}\right| \leqslant \Delta R=R / N$. The symmetry of expression (8) leads to cosine terms for the translational freedom, and the whole sum is real. Just focusing on these cosine terms leads to the well known Fourier transformation of independent solid spheres

$$
\begin{aligned}
2\langle\cos & {\left.\left[\vec{Q}\left(\vec{v}_{2}+\cdots+\vec{v}_{j}\right)\right]\right\rangle_{v_{2}, \ldots, v_{j}} } \\
& =\left\langle\exp \left[i \vec{Q}\left(\vec{v}_{2}+\cdots+\vec{v}_{j}\right)\right]\right. \\
& \left.+\exp \left[-i \vec{Q}\left(\vec{v}_{2}+\cdots+\vec{v}_{j}\right)\right]\right\rangle_{v_{2}, \ldots, v_{j}}=2[K(Q)]^{j-1} .
\end{aligned}
$$

The average splits into a product of independent statistics of independent random walk steps. Each step is limited by a sphere of radius $\Delta R$, and thus the individual Fourier transformation $K(Q)$ of a single solid sphere is obtained:

$$
K(Q)=3 \frac{\sin (Q \Delta R)-Q \Delta R \cos (Q \Delta R)}{(Q \Delta R)^{3}} .
$$

In a refined description one might take the bilayer thickness $\Delta$ into account for the maximum shift vector, i.e., $\Delta R$ $=R / N-\Delta$. Even more sophisticated statistics might take the thermodynamics of charged bilayers into account for instance. Another simple approach might describe the internal distribution of shift vectors by a Gaussian curve. Throughout this paper we stick to the steric interpretation of $K(Q)$, and we will get valuable information as we will see in the discussion part. Replacing the cosine terms in Eq. (8) by the powers of $K(Q)$ leads to a quite simple formula, which in matrix representation reads

$$
\begin{aligned}
S(Q)= & \frac{N}{\frac{1}{3}\left(N+\frac{1}{2}\right)(N+1)}\left(F_{1}, F_{2}, \ldots, F_{N}\right) \\
& \times\left(\begin{array}{ccccc}
1 & K^{1} & K^{2} & \cdots & K^{N-1} \\
K^{1} & 1 & K^{1} & & \vdots \\
K^{2} & K^{1} & 1 & & K^{2} \\
\vdots & & & \ddots & K^{1} \\
K^{N-1} & \cdots & K^{2} & K^{1} & 1
\end{array}\right)\left(\begin{array}{c}
F_{1} \\
F_{2} \\
\vdots \\
F_{N}
\end{array}\right) .
\end{aligned}
$$

This formula contains all essential information mathematically. Nonetheless, it is possible to give a closed expression for the whole sum, where the explicit expressions of the single shells [Eq. (6)] are taken into account. The closed expression contains only 31 terms. The advantage becomes obvious since for 100 shells Eq. (11) would end up with $10^{4}$ addends. These many addends need more time for the calculation and the error of oscillating terms is amplified by the number of addends. However, the full closed expression is quite lengthy and does not give any further information by visual inspection; so it will be given in the Appendix.

The idea of Eq. (11) was also used in the case of linear polymer chains [24]. Then all individual particles (mono- mers) are pointlike, and the displacement statistics could be simplified by a Gaussian distribution.

In some cases Eq. (11) can be discussed explicitly without too lengthy terms. The two cases $K=1$ and $K=0$ are special by two meanings: On the one hand the shorter formulations have a higher accuracy than the lengthy expression of the Appendix. If $K(Q)$ comes close to either 1 or 0 the following short expressions are used. On the other hand a constant function $K(Q)$ has some physical meaning.

The special case $K(Q)=0$ const means that there is no constructive interference of all shells, i.e., all shells scatter independently. The actually constant function $K(Q)$ might not appear so interesting, but for large $Q$ the function $K(Q)$ of Eq. (10) always tends to 0. This means that on small length scales the surface properties of the shells (as we will see below) are always seen as an independent superposition of all contributions. On small length scales the correlations of larger scales are lost. The special case $K=0$ of Eq. (11) reads

$$
\begin{aligned}
S(q= & Q R / N)=\frac{1}{N^{2} q^{2}}\left\{\frac{1}{2}+\frac{3}{4 N\left(N+\frac{1}{2}\right)(N+1)}\right. \\
& \times\left[\cos [2 q(N+1)]\left(-\frac{N+1}{\sin ^{2} q}+(N+1)^{2}\right)\right. \\
& +\sin [2 q(N+1)] \\
& \left.\left.\times\left(\frac{1}{2 \sin ^{2} q}-(N+1)^{2}\right) / \tan q\right]\right\} .
\end{aligned}
$$

The special case $K(Q)=1$ const means that all shells are exactly concentric and all shell contributions show interference. Again, this special case might look rather specific, but for low scattering angles $K(Q)$ always tends to 1 . This means that on large length scales the MLV always looks the same whether there are shifts of the centres or there are not. On large length scales the Guinier law is observed and only the mass of the vesicle matters. The special case $K=1$ of Eq. (11) reads

$$
\begin{aligned}
S(q= & Q R / N)=\frac{3}{N^{3}\left(N+\frac{1}{2}\right)(N+1)} \\
& \times\left(-\frac{1}{2} \cos \left[q\left(N+\frac{1}{2}\right)\right]\left(N+\frac{1}{2}\right)+\frac{1}{4} \sin \right. \\
& \left.\times\left[q\left(N+\frac{1}{2}\right)\right] / \tan \left(\frac{1}{2} q\right)\right)^{2} /\left[q \sin \left(\frac{1}{2} q\right)\right]^{2} .
\end{aligned}
$$

For numerical reasons the limits are essential, when Eqs. (12) and (13) are good approximations of Eq. (11). From a taylor expansion at $Q=0$ we derived the following handy conditions: 


$$
\begin{gathered}
|K|<10^{-3} \frac{1}{2} \frac{6 N^{5}+15 N^{4}+10 N^{3}-N}{6 N^{5}-10 N^{3}+4 N} \\
|K-1|<10^{-3} \frac{420}{36} \frac{4 N^{6}+12 N^{5}+13 N^{4}+6 N^{3}+N^{2}}{10 N^{7}+36 N^{6}+21 N^{5}-35 N^{4}-35 N^{3}+4 N} .
\end{gathered}
$$

With these equations the analytical options are exhausted. But numerical calculations will shine more light on the complicated relations derived so far.

\section{FORM FACTORS OF BILAYERS AND MACROSCOPIC CROSS SECTION}

While the mathematical problems lie in the structure factor, a simple form factor shall be discussed and the model macroscopic cross section shall be given. The simplest structure bases on a homogenous bilayer which might be a good description for "simple" surfactants in heavy water. As a first approximation the neutron scattering length density of heavy water generates a clear contrast to protonated surfactants and internal inhomogeneities shall be neglected. The basics of the form factor are developed in concert with Eqs. (4) and (5), and for the present assumptions it reads

$$
F(Q)=\left(\frac{\sin (Q \Delta / 2)}{Q \Delta / 2}\right)^{2}
$$

The splitting of structure and form factors holds well if the bilayer thickness $\Delta$ is small compared to all radii of all shells $\Delta \ll R j / N$ (for all $j$ ). This is especially important for the smallest radius, and so the condition reads $\Delta \ll R / N$. This means that all single shells should not intersect. The connected macroscopic cross section reads now

$$
\frac{d \Sigma}{d \Omega}(Q)=(\Delta \rho)^{2} \phi V_{\text {oshell }} S(Q) F(Q) .
$$

The current normalization of the structure factor was done in this way that it is proportional to the sum of all fractions of surfaces of the inner shells [see Eq. (8)]. So the remaining volume of the outer shell appears $V_{\text {oshell }}=4 \pi R^{2} \Delta$. The whole appearance of Eq. (17) is rather simple, but all contributions to the forward scattering are clearly addressed.

For more realistic form factors more detailed models can be made (as in Refs. [13,22]). For surfactants one could assume scattering length density profiles with clearly distinct values in separate regions. If the surfactant molecules stick out of the bilayer with a distribution of lengths some smearing of the scattering length density profile has to be assumed [25]. For polymers forming a hairy bilayer, the scattering length density of the polymer brush had to be modeled as well. The current paper aims at the major properties of the structure factor and material dependent form factors are left open for individual modeling.

\section{DISCUSSION}

The numerical calculations will vary some parameters of this model. First, the number of shells $N$ is varied, while

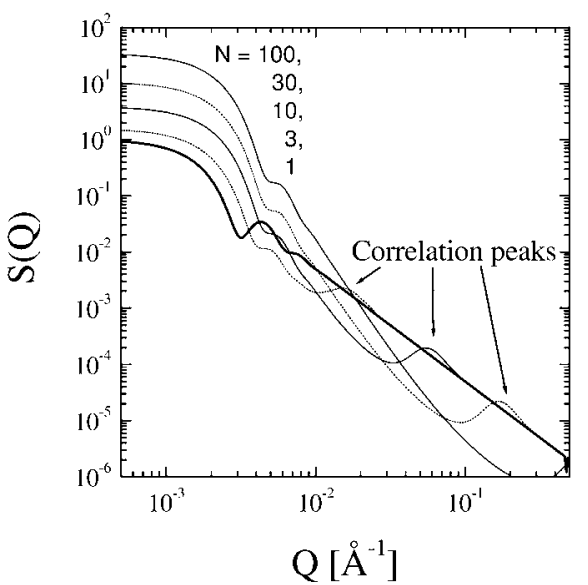

FIG. 2. Theoretical scattering for a multilamellar vesicle with different numbers of layers $N$. For simplicity the outer radius $R$ $=1000 \AA$ was kept constant. A $15 \%$ smearing of the intensities was allowed to simulate polydispersity and instrument resolution. (Slightly modified, taken from Ref. [2].)

secondly the disorder-connected with the maximum shift $\Delta R$-is varied. For these two examples the maximum radius $R$ was kept constant, but a reasonable polydispersity of $R$ was simulated. After these two series of parameter variations the development stage of this model is discussed.

The first series of structure factors is shown in Fig. 2, where the $K=1$ const limit for exactly concentric shells is calculated. A smearing of $15 \%$ was allowed, which we simulated by a polydispersity of the radius $R$. Small smearing of length scales is realistic, since a scattering experiment has a final resolution and a polydispersity of $R$ is present. The polydispersity of $N$ was not regarded so far. The series presents results for $N=1,3,10,30$, and 100 shells. The radius was chosen to be $R=1000 \AA$. The thick line shows a unilamellar vesicle $(N=1)$. At low scattering angles $(Q$ $<0.002 \AA^{-1}$ ) the Gunier range can be observed. The structure factor is normalized in this way, that the low $Q$ limit is unity. At slightly higher $Q$ (up to $0.01 \AA^{-1}$ ) some oscillations appear due to the finite size (or better: sharp boundaries) of the vesicle. At higher $Q\left(Q>0.01 \AA^{-1}\right)$ the power law of surfaces becomes dominant. For the vesicles with more shells, the outer radius $R$ is kept constant. The low $Q$ limit $\left(Q<0.003 \AA^{-1}\right)$ keeps track of the material inside the vesicle. At slightly higher $Q\left(Q<0.007 \AA^{-1}\right)$ there are always oscillations, but the first minimum is shifted to higher $Q$ which is expected when changing from a hollow to a compact sphere. At slightly higher $Q \quad(Q<0.01,0.03$, $\left.0.1,0.3 \AA^{-1}\right)$ a power-law region appears which tells about the compactness of the vesicle. It changes from $Q^{-2}$ for surfaces to $Q^{-4}$ for compact spheres. At a $Q$ of roughly $2 \pi N / R$ a correlation peak appears, which scales with the distance between the layers. At even higher $Q$ the surface of single layers becomes visible. Here, all functions meet again, since the concentration of material was assumed to be identical. Since we describe the structure factor at this moment a simple property of surfaces is seen here.

The compactness exponent $\alpha$ of the first power law $\mathrm{Q}^{\alpha}$ of this model is shown in Fig. 3 as a function of the shell 


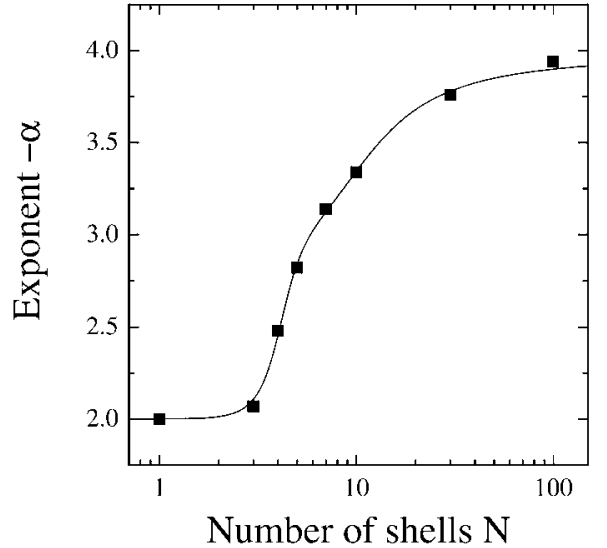

FIG. 3. The slope of a $\log -\log$ plot $S(Q)$ versus $Q$ in the first power law region is a measure for the compactness of the multilamellar vesicle. For a small number of shells $N$ the surface properties dominate, while at large number of shells the MLV shows compact volume properties. The line is a guide for the eye.

number. Between 3 and $\sim 30$ shells the exponent is considerably increasing from 2 to roughly 4 , which should be the interesting range for experiments. This graph might be used to compare experimentally obtained exponents $\alpha$ with the shell number. Already the Guinier region of scattering tells about the material within a single vesicle, and therefore can give an estimation about the shell number $N$. This experiment can be done by small angle light scattering.

The second set of calculations shall demonstrate the influence of disorder, which was simulated by a maximum shift vector $\Delta R$ for each shell (Fig. 4). The outer radius $R$ $=1000 \AA$, and the number of shells $N=10$ was kept constant. $\Delta R$ was varied between $0.1,60$, and $100 \AA$. For a given maximum displacement $\Delta R$ the larger displacements $|\vec{v}|$ are favored since the probability increases with $|\vec{v}|^{2}$. The latter

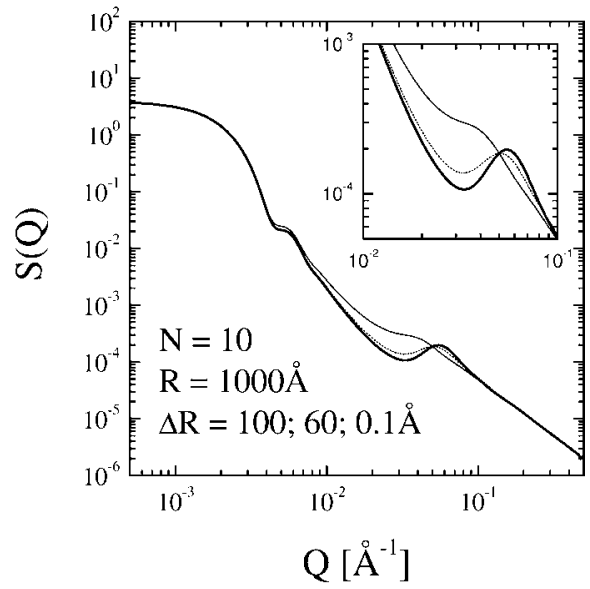

FIG. 4. Theoretical scattering for a multilamellar vesicle with different degrees of disorder. The maximum shift radius varies from 0.1 over 60 to $100 \AA$ (thick, dotted, thin line), which is the theoretical maximum, because the shell-to-shell distance is $100 \AA$. The outer radius $R=1000 \AA$, and the number of shells $N=10$ are kept constant. Again, as in Fig. 2, a 15\% smearing was applied. value $\Delta R=100 \AA$ is the physical maximum, since the shells would intersect otherwise. One observes that the peak moves to lower scattering angles and finally ends up as a shoulder between the two power laws connected to the compactness of the vesicle and the surface properties of single shells. With large disorder many parts of the shells merge together and the whole vesicle looks more open (as already indicated by Fig. 1), which is reflected by a smaller slope of the compactness power law and a smaller correlation peak scattering vector. The $15 \%$ smearing of the radius parameter does not considerably affect the sharpness of the correlation peak. This means that low degrees of disorder (small $\Delta R$ ) do not change the appearance of the correlation peak, which makes the model rather predictive. As a practical relative maximum sharpness of this correlation peak we obtain a FWHM of $35 \%$ for $N=10$ and $\Delta R=0.1 \AA$ and a smearing of $2 \%$ only, which should be a criterion for the applicability of this model.

If all length scales of the model $(R, \Delta R)$ are scaled up, the principal shape of the scattering curves does not change. This is interesting for hydration phenomena, for instance. Nonetheless, the degree of fluctuations could change.

The first stage of concentric shells was already published in Ref. [2]. The next higher level including translational disorder (displacements) is presented in this article. Since the undulations of the shells are neglected, this model corresponds to extremely rigid membranes (The existence of the inner sharply curved shells is a question, but the scattering contribution of these shells is a minor one.) These models are initial models, before a complete picture of the scattering of MLVs will be obtained in the near future. A next higher step of modeling could include independent undulations of the shells by the Lisy model $[16,17]$, which corresponds to rather well defined centres of the shells still which fluctuate weakly due to a moderately high bending rigidity. If the membrane rigidity is even lower, the displacements are ill defined and the local strong fluctuations dominate the picture. In principle, several stages of description have been passed through for lamellar phases, as discussed by Ref. [15]. The rigidity of the membranes is titles "hard" and "soft," while the positional definition of the membranes is connected with the smectic penetration length, which is large for well defined positions ("hard smectic order") and vice versa ("soft smectic order"). The next step to (hard smectic order) for MLVs should be an easy one with the Lisy model.

The next higher steps of modeling will change the peak shape and position. Intuitively the peak will get broader due to the fluctuations, and thus the present model gives an upper limit for the peak sharpness. Similarly, it is unlikely that a higher order peak appears with stronger fluctuations. The intensity at smaller scattering vectors could increase due to the fluctuations, and so the exponent alpha could be modified, and the clear information about the compactness could be blurred.

\section{SUMMARY}

A scattering model for multilamellar vesicles is developed covering many length scales from the whole particle down to 
the bilayer. The most important properties are derived from the structure factor idealizing the shells as infinitely thin. The parameters of the model are the radius of the vesicle $R$, the number of shells $N$, and a maximum shift $\Delta R$. The model assumes an arithmetic series of radii for the spherical shells. Due to the steric hindrance of the shells, the maximum shift is $\Delta R \leqslant R / N$. The centers of the shells describe a random walk.

The following $Q$ ranges are found for this model sorted from large to small length scales:

The Guinier law describes the whole vesicle. Initial oscillations appear due to the sharp boundaries of the particle. The first power law region is connected to the compactness of the vesicle. It varies continuously from $Q^{-2}$ for surfaces to $Q^{-4}$ for compact bodies and is connected with the number of shells $N$. The correlation peak describes the spacing of the bilayers. A large maximum shift parameter $\Delta R$ reduces the sharpness of the peak and shifts the peak to smaller scattering vectors. No second order correlation peaks are described. The second power law accounts for the surface properties of the bilayer. The whole model is predictive for the first power law describing the compactness of the vesicles and for the correlation peak which has a maximum sharpness rather independent of the shell number $N$.

The transition of the compactness exponent between -2 and -4 was successfully observed by experiments in parallel [2]. Experiments covering the whole $Q$ range remain to be conducted. Especially then the three essential parameters $R$, $N$, and $\Delta R$ can be determined independently. The assumption of dilution would be rather important for first tests.

\section{APPENDIX}

The complex formula of Eq. (11) does not look to have an explicit solution for all arbitrary $N$, but algebraic programs can solve this problem by rewriting the sine of Eq. (6) to complex exponentials. Then the whole Eq. (11) has the form of four terms similar to $\Sigma_{i, j} i a^{i} j a^{ \pm j} K^{|j-i|}$. Throughout the appendix the indices $i$ and $j$ are integers. The denominator of the solution reads

$$
\begin{aligned}
& D(q)=\left[-6\left(K^{2}-1\right)\left(K^{2}+1\right)\left(K^{4}+5 K^{2}+1\right) n^{2}+\left(-6 K^{8}-12 K^{6}+48 K^{4}+48 K^{2}+6\right) n+\left(3 K^{8}+36 K^{6}+24 K^{4}-18 K^{2}\right.\right. \\
& -3)] \sin [q(2 N+1)]+\left[\left(3 K^{8}-12 K^{6}-45 K^{4}-24 K^{2}-3\right) n^{2}+\left(6 K^{8}-12 K^{6}-72 K^{4}-48 K^{2}-6\right) n+\left(3 K^{8}+18 K^{6}\right.\right. \\
& \left.\left.-24 K^{4}-36 K^{2}-3\right)\right] \sin [q(2 N-1)]+\left(\left(3 K^{8}+24 K^{6}+45 K^{4}+12 K^{2}-3\right) n^{2}+6 K^{2}\left(3 K^{2}+2\right) n+3 K^{2}\left(4 K^{4}-K^{2}\right.\right. \\
& -6)) \cdot \sin (q(2 N+3))+\left(18 K^{4}\left(K^{2}+1\right) n^{2}+6 K^{2}\left(6 K^{4}+3 K^{2}-2\right) n+3 K^{2}\left(6 K^{4}+K^{2}-4\right)\right) \cdot \sin (q(2 N-3)) \\
& +\left(-18 K^{2}\left(K^{2}+1\right) n^{2}-6 K^{4}\left(2 K^{2}+3\right) n-3 K^{4}\right) \cdot \sin (q(2 N+5))+\left(3 K^{4} n^{2}+6 K^{4} n+3 K^{4}\right) \cdot \sin (q(2 N-5)) \\
& +\left(-3 K^{4} n^{2}\right) \cdot \sin (q(2 N+7))+\left[6 K^{3}\left(3 K^{4}+10 K^{2}+5\right) n^{2}+6 K^{3}\left(3 K^{4}+8 K^{2}+3\right) n-12 K\left(K^{2}-1\right)\left(K^{4}+3 K^{2}\right.\right. \\
& +1)] \sin (q 2 N)+\left[K\left(-12 K^{6}-12 K^{4}+12 K^{2}+6\right) n^{2}-6 K\left(2 K^{2}+3\right)\left(2 K^{4}-2 K^{2}-1\right) n+K\left(-12 K^{6}-12 K^{4}+36 K^{2}\right.\right. \\
& +12)] \sin [q(2 N-2)]+\left[K\left(-30 K^{4}-60 K^{2}-18\right) n^{2}+K\left(-42 K^{4}-72 K^{2}-18\right) n+K\left(-12 K^{6}-36 K^{4}+12 K^{2}\right.\right. \\
& +12)] \sin [q(2 N+2)]+\left[-6 K^{3}\left(2 K^{2}+1\right) n^{2}-6 K^{3}\left(4 K^{2}+1\right) n-12 K^{5}\right] \sin [q(2 N-4)]+\left[-6 K\left(K^{6}+2 K^{4}-2 K^{2}-2\right) n^{2}\right. \\
& \left.+6 K^{3}\left(K^{4}+4 K^{2}+2\right) n+12 K^{3}\right] \sin [q(2 N+4)]+\left[6 K^{3}\left(K^{2}+2\right) n^{2}+6 K^{5} n\right] \sin [q(2 N+6)]+\left[6 ( K ^ { 2 } - 1 ) ( K ^ { 2 } + 1 ) \left(K^{4}\right.\right. \\
& \left.+4 K^{2}+1\right) n^{3}+3\left(K^{4}-4 K^{2}-3\right)\left(3 K^{4}+8 K^{2}+1\right) n^{2}+\left(3 K^{8}-36 K^{6}-120 K^{4}-60 K^{2}-3\right) n+42 K^{2}\left(K^{4}-4 K^{2}\right. \\
& +1)] \sin (q)+\left[-2\left(K^{2}-1\right)\left(K^{2}+1\right)\left(K^{4}-K^{2}+1\right) n^{3}+\left(-3 K^{8}+9 K^{6}+3 K^{2}+3\right) n^{2}+\left(-K^{8}+7 K^{6}+5 K^{2}+1\right) n+6 K^{2}\right. \\
& \left.\left(-4 K^{4}+11 K^{2}-4\right)\right] \sin (3 q)+\left[-6 K^{2}\left(K^{2}-1\right)\left(K^{2}+1\right) n^{3}-3 K^{2}\left(K^{4}-8 K^{2}-5\right) n^{2}+3 K^{2}\left(K^{4}+8 K^{2}+3\right) n+6 K^{2}\left(K^{4}\right.\right. \\
& \left.\left.-K^{2}+1\right)\right] \sin (5 q)+\left[-6 K\left(K^{2}-1\right)\left(2 K^{2}+1\right)\left(K^{2}+2\right) n^{3}+K\left(-12 K^{6}+48 K^{4}+102 K^{2}+24\right) n^{2}+K\left(66 K^{4}+84 K^{2}\right.\right. \\
& \left.+12) n+24 K^{3}\left(K^{2}+1\right)\right] \sin (2 q)+\left[6 K\left(K^{2}-1\right)\left(K^{2}+1\right)^{2} n^{3}+6 K\left(K^{2}+1\right)\left(K^{4}-5 K^{2}-2\right) n^{2}-6 K\left(K^{2}+1\right)\left(5 K^{2}+1\right) n\right. \\
& \left.-12 K^{3}\left(K^{2}+1\right)\right] \sin (4 q)+\left[2 K^{3}\left(K^{2}-1\right) n^{3}-6 K^{3} n^{2}-2 K^{3}\left(K^{2}+2\right) n\right] \sin (6 q)+K^{N+2}\left(K^{2}-1\right)\{\sin (q N) K[-72 \\
& \left.-12 N\left(3 K^{2}+4\right)\right]+\sin [q(N-1)]\left[12\left(3 K^{2}-2\right)-12 N\left(K^{2}+2\right)\right]+\sin [q(N+1)]\left[-12\left(2 K^{2}-3\right)+12 N\left(4 K^{2}+3\right)\right] \\
& +\sin [q(N-2)] K\left[48+6 N\left(4 K^{2}+7\right)\right]+\sin [q(N+2)] K\left[48+12 N\left(2 K^{2}+1\right)\right]+\sin [q(N-3)]\left[-6\left(4 K^{2}-1\right)\right. \\
& \left.-6 N\left(2 K^{2}-1\right)\right]+\sin [q(N+3)]\left[6\left(K^{2}-4\right)-6 N\left(7 K^{2}+4\right)\right]+\sin [q(N-4)] K\left[-12-6 N\left(K^{2}+2\right)\right]+\sin [q(N+4)] \\
& \left.\times K\left[-12-6 N\left(K^{2}-2\right)\right]+\sin [q(N-5)] K^{2}(6+6 N)+\sin [q(N+5)]\left[6+6 N\left(2 K^{2}+1\right)\right]+\sin [q(N+6)] K(-6 N)\right\} \text {. }
\end{aligned}
$$


Please remember that $q=Q R / N$, and the function $K$ depends on $Q$ as well. Together with the numerator the whole solution reads

$$
\begin{aligned}
S(q= & Q R / N)=\frac{3}{N\left(N+\frac{1}{2}\right)(N+1)} D(q) /\left\{-48 N^{2} \sin ^{3}(q)\right. \\
& \left.\times\left[K^{2}+1-2 K \cos (q)\right]^{4} q^{2}\right\} .
\end{aligned}
$$

The form of Eqs. (A1) and (A2) does not evaluate well for $K \approx 1, K \approx 0$, and non-natural $N$. The limits of $K=0,1$ are described by Eqs. (12) and (13). The acceptance of natural $N$ is correct in a physical sense, but for fitting programs one better likes to allow for continuous changes of $N$. For nonnatural $N$ one could interpolate between the neighbouring naturals. If one includes the polydispersity of $N$, a summation over several $N$ has to be done anyways.

The explicit formula of Eqs. (A1) and (A2) has 31 oscillating sine terms. These terms partially cancel out, and it is numerically hard to obtain the leading term. For instance, the limit for small $Q$ is a constant (Guinier region). For small $Q$ the numerator of Eq. (A2) is proportional to $Q^{5}$ if $K<1$. This means that for a Taylor series of the denominator [Eq. (A1)] the first coefficients of the powers $Q^{1}$ and $Q^{3}$ cancel out, and the coefficient of the $Q^{5}$ power leads to a finite forward scattering. For higher $Q$ the whole situation becomes not arranged clearly. The numerical solution is a Taylor expansion of the sine terms in the usual manner with the trick of adding all terms in a special way. The denominator has the following form:

$$
D=\sum_{i} A_{i} \sin \left(B_{i}\right) .
$$

The index $i$ is an integer number. Then a Taylor series describes the sine and cosine as expansions around natural multiples of $\pi / 2$ :

$$
D=\sum_{j} \sum_{i} \alpha(i, j) A_{i} \frac{1}{j !}\left(B_{i}-\beta_{i} \pi / 2\right)^{j} .
$$

The number $\beta_{i}=\left[B_{i} /(\pi / 2)\right]$ tells about the position of the Taylor expansion, where the bracket $[x]$ yields the closest integer number for a real $x$. The coefficient $\alpha(i, j)$ defines the sign and the consideration of a coefficient $\mathrm{A}_{i} / j$ !. In a simple way this coefficient can be written as

$$
\alpha(i, j)=\sin \left[\pi / 2\left(\beta_{i}+j\right)\right],
$$

but a numerically quicker treatment is possible. The trick of this summation is to carry out the inner sum over $i$ first, and the other sum over $j$ afterwards. This makes sure, that coefficients with the same small $j$ can cancel out first before they are added up with terms of larger $j$. This trick is not precise for extremely small $Q$, but usually $K$ tends to 1 then, and the more accurate formula of Eq. (13) takes over.
[1] S. Segota and D. Tezak, Adv. Colloid Interface Sci. 121, 51 (2006).

[2] H. Iatrou, H. Frielinghaus, S. Nevanpää, N. Ferderigos, O. Ikkala, D. Richter, J. Mays, and N. Hadjichristidis, Biomacromolecules 8, 2173 (2007).

[3] W. Z. Helfrich, Z. Naturforsch. A 33, 305 (1978).

[4] L. Ramos, D. Roux, P. D. Olmsted, and M. E. Cates, Europhys. Lett. 66, 888 (2004).

[5] O. Regev and F. Guillemet, Langmuir 15, 4357 (1999).

[6] K. Watanabe, Y. Nakama, T. Yanaki, and H. Hoffmann, Langmuir 17, 7219 (2001).

[7] A. Radulescu, D. Schwahn, J. Stellbrink, E. Kentzinger, M. Heiderich, D. Richter, and L. Fetters, Macromolecules 39, 6142 (2006).

[8] J. Oberdisse, C. Couve, J. Appell, J. F. Berret, C. Ligoure, and G. Porte, Langmuir 12, 1212 (1996).

[9] R. Beck, M. Gradzielski, K. Horbaschek, S. Sakhawat Shah, H. Hoffmann, and P. Strunz, Adv. Colloid Interface Sci. 221, 200 (2000).

[10] T. M. Weiss, T. Narayanan, C. Wolf, M. Gradzielski, P. Panine, S. Finet, and W. I. Helsby, Phys. Rev. Lett. 94, 038303 (2005).

[11] M. P. Nieh, T. A. Harroun, V. A. Raghunathan, C. J. Glinka, and J. Katsaras, Phys. Rev. Lett. 91, 158105 (2003).
[12] M. A. Caillé, C. R. Acad. Sci. B 274, 891 (1972).

[13] K. D. Goecking and M. Monkenbusch, Europhys. Lett. 43, 135 (1998).

[14] L. Gunther, Y. Imry, and J. Lajzerowicz, Phys. Rev. A 22, 1733 (1980).

[15] F. Castro-Roman, L. Porcar, G. Porte, and C. Ligoure, Eur. Phys. J. E 18, 259 (2005).

[16] V. Lisy, B. Brutovsky, and A. V. Zatovsky, Phys. Rev. E 58, 7598 (1998).

[17] V. Lisy, B. Brutovsky, and P. Miskovsky, Physica B 266, 300 (1999).

[18] S. A. Safran, J. Chem. Phys. 78, 2073 (1983).

[19] S. A. Safran, Phys. Rev. A 43, 2903 (1991).

[20] R. Abdel-Rahem and H. Hoffmann, Rheol. Acta 45, 781 (2006).

[21] T. Hellweg, A. Brulet, A. Lapp, D. Robertson, and J. Kötz, Phys. Chem. Chem. Phys. 4, 2612 (2002).

[22] F. Nallet, R. Laversanne, and D. Roux, J. Phys. II 3, 487 (1993).

[23] J. S. Pedersen, Adv. Colloid Interface Sci. 70, 171 (1997).

[24] J. S. Higgins and H. C. Benoît, Polymers and Neutron Scattering (Clarendon Press, Oxford, 1994).

[25] R. Strey, Colloid Polym. Sci. 272, 1005 (1994). 